Pacific Journal of Mathematics

THE BOUNDARY BEHAVIOUR OF HARMONIC UNIVALENT
MAPS Yusuf ABU-MUHANNA AND ABDALLAH KhaLID LYZZAI 


\title{
THE BOUNDARY BEHAVIOUR OF HARMONIC UNIVALENT MAPS
}

\author{
Yusuf Abu-Muhanna AND Abdallah Lyzzaik
}

Let $D$ denote the open unit disc in the complex plane and $f=h+\bar{g}$ a complex-valued, harmonic, univalent and orientation preserving map in $D$, where $h$ and $g$ are analytic in $D$. We show that $g, h \in H^{\lambda}$ and $f \in h^{\lambda}$ for some $\lambda>0$, where $H^{\lambda}\left(h^{\lambda}\right)$ is the Hardy space of order $\lambda$ for analytic (harmonic) functions. We also study the correspondence under $f$ between $\partial D$ (boundary of $D$ ) and the prime ends of $f(D)$.

1. Introduction. Let $D$ denote the open unit disk and $S_{H}$ denote the class of all complex valued, harmonic, orientation-preserving, univalent functions $f$ in $D$ normalized by

$$
f(0)=0 \text { and } f_{z}(0)=1 .
$$

Each $f \in S_{H}$ can be expressed as

$$
f=h+\bar{g}
$$

where $h=z+\sum_{n=2}^{\infty} a_{n} z^{n}$ and $g=\sum_{n=1}^{\infty} b_{n} z^{n}$ are analytic in $D$. Clunie and Sheil-Small, [3], studied $S_{H}$ together with some geometric subclasses of $S_{H}$. They proved, among other results, that $S_{H}$ is normal with respect to the topology of uniform convergence on compact subsets of $D$. In this paper, we study the aspect of boundary behaviour of functions in $S_{H}$. We point out that, although some of our results are stated for $f \in S_{H}$, conditions (1) are not needed.

It is known that, when $f \in S_{H}$ is also analytic, the length of the image of the radius $\left[0, e^{i \theta}\right], \int_{0}^{1}\left|f^{\prime}\left(r e^{i \theta}\right)\right| d r$, is finite for all $\theta$ except for a set of logarithmic capacity zero [8, p. 341]. It is also known that $f \in H^{\lambda}, 0<\lambda<\frac{1}{2}$, where $H^{\lambda}$ is the Hardy space of analytic functions of order $\lambda[8$, p. 127], [5, p. 61]. In $\S 2$, we prove that the length of the image of the radius $\left[0, e^{i \theta}\right]$ under $f \in S_{H}, \int_{0}^{1}\left|\frac{\partial f}{\partial r}\left(r e^{i \theta}\right)\right|$, is finite for almost all $\theta$. We also prove that $h, g \in H^{\lambda}$ and $f \in h^{\lambda}$ for some $\lambda$ small, where $h^{\lambda}$ is the Hardy space of harmonic functions of order $\lambda[4$, p. 2]. Furthermore, as a corollary, we conclude that the radial limits $\hat{h}\left(e^{i \theta}\right)=\lim _{r \rightarrow 1^{-}} h\left(r e^{i \theta}\right), \hat{g}\left(e^{i \theta}\right)=\lim _{r \rightarrow 1^{-}} g\left(r e^{i \theta}\right)$ and $\hat{f}\left(e^{i \theta}\right)=\lim _{r \rightarrow 1^{-}} f\left(r e^{i \theta}\right)$ exist for almost all $\theta[4$, pp. 15-17]. 
One of the many differences between harmonic univalent maps and conformal maps is that a harmonic univalent map can be constant on an interval of the boundary of $D, \partial D$. In $\S 3$, we show that if $f \in S_{H}$ and $f$ is constant on an interval then the interval "corresponds" to a prime-end satisfying a wedge condition (see Theorem 3 ). In addition, we obtain some corollaries.

In $\S 4$, we study the correspondence, under $f \in S_{H}$, between points of $\partial D$ and the prime-ends of $f(D)$ (see Theorems 6,7).

2. Radial limits. First, we have the following two lemmas.

Lemma 1. Let $f=h+\bar{g} \in S_{H}$. Then $\log h^{\prime}$ is a Bloch function, that is,

$$
\left|\frac{h^{\prime \prime}(z)}{h^{\prime}(z)}\right| \leq \frac{c}{1-r} \quad\left(z=r e^{i \theta}\right)
$$

for some absolute constant $c$.

Proof. For fixed $\zeta \in D$ consider the function

$$
F(z)=\frac{f\left(\frac{z+\zeta}{1+\bar{\zeta} z}\right)-f(\zeta)}{\left(1-|\zeta|^{2}\right) h^{\prime}(\zeta)} .
$$

Then $F \in S_{H}$, and we can write

$$
F(z)=H(z)+\bar{G}(z)
$$

where

$$
H(z)=z+\sum_{n=2}^{\infty} A_{n} z^{n} .
$$

By direct computation we obtain

$$
2 A=\left(1-|\zeta|^{2}\right) \frac{h^{\prime \prime}(\zeta)}{h^{\prime}(\zeta)}-2 \bar{\zeta}
$$

Since $A=\sup _{S_{H}}\left|a_{2}\right|$ is finite [3],

$$
\left.|1-| \zeta\right|^{2}|| \frac{h^{\prime \prime}(\zeta)}{h^{\prime}(\zeta)} \mid \leq 2 A+2=c
$$

and the proof is complete since $\zeta$ is arbitrary.

LEMMA 2. Let $h$ be an analytic and locally univalent function in $D$ satisfying inequality (2). If $\alpha>0$, then

$$
\lim _{r \rightarrow 1^{-}}(1-r)^{\alpha} h^{\prime}\left(r e^{i \theta}\right)=0
$$

for almost all $\theta$. 
This is a result of J. Clunie and T. MacGregor [2], [7, p. 73]. As a consequence of Lemma 2 we have the following theorem.

Theorem 1. Let $f=h+\bar{g} \in S_{H}$. Then the integrals

$$
\int_{0}^{1}\left|h^{\prime}\left(r e^{i \theta}\right)\right| d r, \quad \int_{0}^{1}\left|g^{\prime}\left(r e^{i \theta}\right)\right| d r, \quad \text { and } \quad \int_{0}^{1}\left|\frac{\partial f}{\partial r}\right| d r
$$

converge for almost all $\theta$, and the boundary function $\hat{f}\left(e^{i \theta}\right)$ exists almost everywhere.

Proof. Choose $0<\alpha<1$. Then by Lemma 2,

$$
\lim _{r \rightarrow 1^{-}}(1-r)^{\alpha} h^{\prime}\left(r e^{i \theta}\right)=0
$$

for almost all $\theta$. Hence we have

$$
\mid h^{\prime}\left(r e^{i \theta}\right) \leq \frac{k_{\theta}}{(1-r)^{\alpha}}
$$

for some constant $k_{\theta}$, which gives at once $\int_{0}^{1}\left|h^{\prime}\left(r e^{i \theta}\right)\right| d r<\infty$ for almost all $\theta$. This yields directly the convergence of the other integrals since $\left|g^{\prime}\right|<\left|h^{\prime}\right|$ because $f \in S_{H}$, and

$$
\frac{\partial f}{\partial r}\left(r e^{i \theta}\right)=e^{i \theta} h^{\prime}\left(r e^{i \theta}\right)+\overline{e^{i \theta} g^{\prime}\left(r e^{i \theta}\right)} .
$$

Now observe that $\int_{0}^{1}\left|\frac{\partial f}{\partial r}(r, \theta)\right| d r<\infty$ means that the image curve under $f$ of the radius: $r e^{i \theta}, 0 \leq r<1$ is rectifiable. This evidently implies that $\hat{f}\left(e^{i \theta}\right)$ exists a.e. and the proof is complete.

Our next result states that every normalized harmonic univalent map together with its analytic and coanalytic parts belong to some Hardy spaces which also imply that $\hat{f}\left(e^{i \theta}\right)$ exists almost everywhere. In particular, we have:

Theorem 2. Let $f=h+\bar{g} \in S_{H}$. Then $h, g \in H^{\lambda}$ and $f \in h^{\lambda}$ for every $\lambda, 0<\lambda<1 / c^{2}$, where $c$ is the absolute constant of Lemma 1 .

Proof. First we show that $h \in H^{\lambda}$ for all $\lambda<1 / c^{2}$. For this purpose we show that for $\lambda>0$ the inequality:

$$
\begin{aligned}
I_{\lambda}\left(r, h^{\prime}\right) & =\frac{1}{2 \pi} \int_{-\pi}^{\pi}\left|h^{\prime}\left(r e^{i \theta}\right)\right|^{\lambda} d \theta \\
& \leq 2\left(1+\lambda^{2} c^{2}\right)(1-r)^{-\beta} \quad(0 \leq r \leq 1)
\end{aligned}
$$


where $\beta=\frac{1}{2 \pi}\left(\sqrt{1+4 c^{2} \lambda^{2}}-1\right)<c^{2} \lambda^{2}$ and $c$ is the constant of Lemma 1.

The proof of (3) is an adaptation of that of Lemma 5.3 in [8, pp. 128-129] from which it follows that for $r_{0}<r<1$ and $\lambda>0$,

$$
I_{\lambda}\left(r, h^{\prime}\right) \leq u(r)=K_{1}\left(c, r_{0}, \lambda\right)(1-r)^{-\beta}+K_{2}\left(c, r_{0}, \lambda\right)(1-r)^{\beta+1}
$$

where

$$
\begin{aligned}
K_{1}\left(c, r_{0}, \lambda\right)= & \frac{1+\beta}{1+2 \beta} I_{\lambda}\left(r_{0}, h^{\prime}\right)\left(1-r_{0}\right)^{\beta} \\
& +\frac{1}{1+2 \beta} I_{\lambda}^{\prime}\left(r_{0}, h^{\prime}\right)\left(1-r_{0}\right)^{\beta+1},
\end{aligned}
$$

and

$$
\begin{aligned}
K_{2}\left(c, r_{0}, \lambda\right)= & \frac{\beta}{1+2 \beta} I_{\lambda}\left(r_{0}, h^{\prime}\right)\left(1-r_{0}\right)^{-\beta-1} \\
& -\frac{1}{1+2 \beta} I_{\lambda}^{\prime}\left(r_{0}, h^{\prime}\right)\left(1-r_{0}\right)^{-\beta} .
\end{aligned}
$$

Using Lemma 5.1 [8, pp. 125-126] and Lemma 1, we obtain

$$
\begin{aligned}
\frac{d}{d r}\left[r I_{\lambda}^{\prime}\left(r, h^{\prime}\right)\right] & =\frac{\lambda^{2} r}{2 \pi} \int_{0}^{2 \pi}\left|h^{\prime}\left(r e^{i \theta}\right)\right|^{\lambda}\left|\frac{h^{\prime \prime}\left(r e^{i \theta}\right)}{h^{\prime}\left(r e^{i \theta}\right)}\right|^{2} d \theta \\
& \leq \frac{c^{2} \lambda^{2} r}{(1-r)^{2}} I_{\lambda}\left(r, h^{\prime}\right) .
\end{aligned}
$$

Then

$$
\begin{aligned}
r I_{\lambda}^{\prime}\left(r, h^{\prime}\right) & \leq c^{2} \lambda^{2} \int_{0}^{r} \frac{t}{(1-t)^{2}} I_{\lambda}\left(t, h^{\prime}\right) d t \\
& \leq c^{2} \lambda^{2} I_{\lambda}\left(r, h^{\prime}\right) \int_{0}^{r} \frac{t}{(1-t)^{2}} d t
\end{aligned}
$$

since $I_{\lambda}\left(t, h^{\prime}\right)$ is a nondecreasing function of $t$. Hence

$$
0 \leq I_{\lambda}^{\prime}\left(r_{0}, h^{\prime}\right) \leq \frac{c^{2} \lambda^{2}}{1-r_{0}} I_{\lambda}\left(r_{0}, h^{\prime}\right)
$$

Using this inequality in (5) and (6) we infer

$$
0 \leq K_{1}\left(c, r_{0}, \lambda\right) \leq \frac{1+\beta+c^{2} \lambda^{2}}{1+2 \beta} I_{\lambda}\left(r_{0}, h^{\prime}\right)\left(1-r_{0}\right)^{\beta},
$$

and

$$
0 \leq K_{2}\left(c, r_{0}, \lambda\right) \leq \frac{\beta+c^{2} \lambda^{2}}{1+2 \beta} I_{\lambda}\left(r_{0}, h^{\prime}\right)\left(1-r_{0}\right)^{-\beta-1}
$$


Consequently by using (4) for $r_{0} \leq r<1$ we obtain

$$
\begin{aligned}
I_{\lambda}\left(r, h^{\prime}\right) \leq & {\left[\frac{1+\beta+c^{2} \lambda^{2}}{1+2 \beta} I_{\lambda}\left(r_{0}, h^{\prime}\right)\left(1-r_{0}\right)^{\beta}\right.} \\
& \left.\quad+\frac{\beta+c^{2} \lambda^{2}}{1+2 \beta} I_{\lambda}\left(r_{0}, h^{\prime}\right)\left(1-r_{0}\right)^{-\beta-1}(1-r)^{2 \beta+1}\right](1-r)^{-\beta} \\
\leq 2 & {\left[\frac{1+\beta+c^{2} \lambda^{2}}{1+2 \beta} I_{\lambda}\left(r_{0}, h^{\prime}\right)\left(1-r_{0}\right)^{-\beta-1}\right](1-r)^{-\beta} . }
\end{aligned}
$$

Since $r_{0}$ is arbitrary we can let $r_{0}$ tend to zero. This proves our claim because

$$
1=h^{\prime}(0)=\lim _{r_{0} \rightarrow 0} I_{\lambda}\left(r_{0}, h^{\prime}\right)
$$

and

$$
\frac{1+\beta+c^{2} \lambda^{2}}{1+2 \beta}
$$

attains its maximum value for $\beta \geq 0$ at $\beta=0$.

Now we prove that $h \in H^{\lambda}$ for $0<\lambda<1 / c^{2}$. The tools of proof are the maximal function of $h$ defined by

$$
h^{*}(r, \theta)=\int_{0}^{r} \max _{s \leq t}\left|h^{\prime}\left(s e^{i \theta}\right)\right| d t
$$

which clearly majorises $h$, and the inequality

$$
\begin{aligned}
\int_{-\pi}^{\pi}\left[h^{*}(r, \theta)\right]^{\lambda} d \theta \leq & 2(1-r)^{\lambda} \int_{-\pi}^{\pi}\left(\frac{\partial h^{*}}{\partial r}\right)^{\lambda} d \theta \\
& +2 \lambda \int_{0}^{r}(1-t)^{\lambda-1} d t \int_{-\pi}^{\pi}\left(\frac{\partial h^{*}}{\partial t}\right)^{\lambda} d \theta .
\end{aligned}
$$

Both the maximal function and inequality (7) are due to Hardy and Littlewood [6, pp. 414-415]. So also the fact that there is an absolute constant, $B_{\lambda}$, depending only on $\lambda$, such that

$$
\begin{aligned}
\int_{-\pi}^{\pi}\left(\frac{\partial h^{*}}{\partial r}\right)^{\lambda} d \theta & =\int_{-\pi}^{\pi}\left(\max _{s \leq r}\left|h^{\prime}\left(s e^{i \theta}\right)\right|\right)^{\lambda} d \theta \\
& \leq B_{\lambda} \int_{-\pi}^{\pi}\left|h^{\prime}\left(r e^{i \theta}\right)\right|^{\lambda} d \theta \\
& \leq 2\left(1+c^{2} \lambda^{2}\right) B_{\lambda}(1-r)^{-c^{2} \lambda^{2}}
\end{aligned}
$$

by using (3) and the fact $\beta<c^{2} \lambda^{2}$. Then from (7) we obtain

$$
\begin{aligned}
\int_{-\pi}^{\pi}\left[h^{*}\left(r e^{i \theta}\right)\right]^{\lambda} d \theta \leq & 2\left(1+c^{2} \lambda^{2}\right) B_{\lambda}(1-r)^{\lambda-\lambda^{2} c^{2}} \\
& +4 \lambda\left(1+c^{2} \lambda^{2}\right) B_{\lambda} \int_{0}^{r}(1-t)^{\lambda-c^{2} \lambda^{2}-1} d t .
\end{aligned}
$$


If $0<\lambda<1 / c^{2}$, then $\lambda-\lambda c^{2}>0$ and consequently

$$
\int_{-\pi}^{\pi}\left[h^{*}\left(r e^{i \theta}\right)\right]^{\lambda} d \theta=4 \frac{1+c^{2} \lambda^{2}}{1-c^{2} \lambda} B_{\lambda}+O\left[(1-r)^{\lambda-c^{2} \lambda^{2}}\right]
$$

as $r \rightarrow 1^{-}$. Therefore $h \in H^{\lambda}$ for all $\lambda, 0<\lambda<1 / c^{2}$.

In the same manner, we conclude that $g \in H^{\lambda}$ for the specific values of $\lambda$ since $\left|g^{\prime}\right|<\left|h^{\prime}\right|$. Now $h, g \in H^{\lambda}$ yield directly that $f=h+\bar{g} \in h^{\lambda}$. This completes the proof.

From the above proof we immediately conclude:

Corollary 1. Let $h$ be analytic in D. If $\log h^{\prime}$ is a Bloch function, then $h \in H^{\lambda}$ for some $\lambda$.

3. $\hat{f}$ constant on some arc of $\partial D$. The purpose of this section is to study the boundary behaviour of a function $f \in S_{H}$ along a closed subarc of $\partial D$ on which $f$ extends continuously to a constant function there.

First, we need the following:

Definition 1 . Let $D$ be a simply connected domain in $\mathbb{C}$ with at least two boundary points, $P$ an accessible prime end, and $\left(C_{n}\right)$ a null-chain of $P$. We say that $D$ satisfies the wedge condition at $P$ if there exists a closed circular sector whose vertex belongs to the impression, $I(P)$, otherwise it lies in $D$, and whose sides intersect $C_{n}$ for all large $n$.

We also need the functions $F$ and $\phi$ associated with $f \in S_{H}$ such that

$$
\phi=F^{-1} \circ f
$$

where $F$ is a conformal map from $D$ to $f(D)$ satisfying $F(0)=0$. Hence $\phi$ is an automorphism of $D$ with radial limits existing almost everywhere.

The main theorem of this section can be stated as follows.

Theorem 3. Let $f=h+\bar{g} \in S_{H}$. Suppose that $f$ extends continuously to a nondegenerate subinterval, $J$, of $\partial D$ such that $f(z)=w_{0}$ for all $z \in J$. Then $\phi(J)=\lambda,|\lambda|=1$; and if $P$ is the prime end of $f(D)$ that corresponds to $\lambda$, under $F$, then $f(D)$ satisfies the wedge condition at $P$.

For a definition of a prime-end see [8, pp. 271-277].

The proof of the theorem makes use of the following interesting lemma. 
LemMA 3. Let $f=h+\bar{g}$ be continuous on $\bar{D}$, where $h$ and $g$ are analytic in $D$. Suppose that $f(z)=w_{0}$ for all $z$ belonging to a nondegenerate subinterval $J=\left\{e^{i \theta}: \theta_{1} \leq \theta \leq \theta_{2}\right\}$ of $\partial D$. Then

(a) $f_{\theta}(z) \rightarrow 0$ as $z \rightarrow \zeta$ for every $\zeta \in \operatorname{Int}(J)$.

(b) $h$ and $g$ extend analytically to $\operatorname{Int}(J)$, and

(c) $z h^{\prime}(z)=z g^{\prime}(z)$ for all $z \in \operatorname{Int}(J)$.

Proof. (a) Since $f$ is bounded, we can write

$$
f(z)=\frac{1}{2 \pi} \int_{0}^{2 \pi} \operatorname{Re}\left(\frac{1+z e^{-i t}}{1-z e^{-i t}}\right) f\left(e^{i t}\right) d t, \quad z \in D .
$$

Then

$$
\begin{aligned}
f_{\theta}(z)= & -\frac{w_{0}}{\pi} \operatorname{Im}\left[\int_{\theta_{1}}^{\theta_{2}} \frac{z e^{-i t}}{\left(1-z e^{-i t}\right)^{2}} d t\right] \\
& -\frac{1}{\pi} \int_{\theta_{1}}^{2 \pi+\theta_{1}} \operatorname{Im}\left[\frac{z e^{-i t}}{\left(1-z e^{-i t}\right)^{2}}\right] f\left(e^{i t}\right) d t \\
= & \frac{w_{0}}{\pi} \operatorname{Re}\left[\frac{1}{1-z e^{-i \theta_{2}}}-\frac{1}{1-z e^{-i \theta_{1}}}\right] \\
& -\frac{1}{\pi} \int_{\theta_{2}}^{2 \pi+\theta_{1}} \operatorname{Im}\left[\frac{z e^{-i t}}{\left(1-z e^{-i t}\right)^{2}}\right] f\left(e^{i t}\right) d t,
\end{aligned}
$$

which gives

$$
\begin{aligned}
\left|f_{\theta}(z)\right| \leq & \frac{\left|w_{0}\right|}{\pi}\left|\operatorname{Re}\left[\frac{1}{1-z e^{-i \theta_{2}}}-\frac{1}{1-z e^{-i \theta_{1}}}\right]\right| \\
& +\frac{\|f\|_{\infty}}{\pi} \int_{\theta_{2}}^{2 \pi+\theta_{1}}\left|\operatorname{Im}\left[\frac{z e^{-i t}}{\left(1-z e^{-i t}\right)^{2}}\right]\right| d t .
\end{aligned}
$$

If $\zeta \in \operatorname{Int}(J)$, then for $\varepsilon>0$ there is $\delta>0$ such that

$$
\begin{aligned}
& \left|\operatorname{Im}\left[\frac{z e^{-i t}}{\left(1-z e^{-i t}\right)^{2}}\right]\right|<\varepsilon, \quad \theta_{2} \leq t \leq 2 \pi+\theta_{1}, \quad \text { and } \\
& \left|\operatorname{Re}\left[\frac{1}{1-z e^{-i \theta_{2}}}-\frac{1}{1-z e^{-i \theta_{1}}}\right]\right|<\varepsilon,
\end{aligned}
$$

for all $z,|z-\zeta|<\delta$. Hence

$$
\left|f_{\theta}(z)\right| \leq\left(\frac{\left|w_{0}\right|}{\pi}+2\|f\|_{\infty}\right) \varepsilon
$$

and the proof of (a) is complete. 
(b) It follows from (9) that

$$
\begin{aligned}
h^{\prime}(z)= & f_{z}(z)=\frac{1}{2 \pi} \int_{0}^{2 \pi} \frac{e^{-i t}}{\left(1-z e^{-i t}\right)^{2}} f\left(e^{i t}\right) d t \\
= & \frac{w_{0}}{2 \pi} \int_{\theta_{1}}^{\theta_{2}} \frac{e^{-i t}}{\left(1-z e^{-i t}\right)^{2}} d t+\frac{1}{2 \pi} \int_{\theta_{2}}^{2 \pi+\theta_{1}} \frac{e^{-i t}}{\left(1-z e^{-i t}\right)^{2}} f\left(e^{i t}\right) d t \\
= & \frac{w_{0}}{2}\left[\frac{e^{-i \theta_{1}}-e^{-i \theta_{2}}}{\left(1-z e^{-i \theta_{1}}\right)\left(1-z e^{-i \theta_{2}}\right)}\right] \\
& +\frac{1}{2 \pi} \int_{\theta_{2}}^{2 \pi+\theta_{1}} \frac{e^{-i t}}{\left(1-z e^{-i t}\right)^{2}} f\left(e^{i t}\right) d t .
\end{aligned}
$$

Clearly, each of the latter terms is an analytic function in

$$
\mathbb{C} \backslash(\partial D \backslash \operatorname{Int}(J)) \text {. }
$$

Hence $h^{\prime}$ is an analytic function there, and so $h$ extends to an analytic function in any simply connected domain containing $D \in \operatorname{Int}(J)$.

As for $g$ we have

$$
\begin{aligned}
g^{\prime}(z)= & \bar{f}_{\bar{z}}(z)=\frac{\bar{w}_{0}}{2 \pi i}\left[\frac{e^{-i \theta_{1}}-e^{i \theta_{2}}}{\left(1-z e^{-i \theta_{1}}\right)\left(1-z e^{-i \theta_{2}}\right)}\right] \\
& +\frac{1}{2 \pi} \int_{\theta_{2}}^{2 \pi+\theta_{1}} \frac{e^{-i t}}{\left(1-z e^{-i t}\right)^{2}} \bar{f}\left(e^{i t}\right) d t
\end{aligned}
$$

and arguing as above for $h$, we conclude (b).

(c) This follows immediately from (a) and (b), and the fact that $f_{\theta}(z)=i\left(z h^{\prime}(z)-\overline{z g^{\prime}(z)}\right), z \in D$.

Proof of Theorem. First we claim that there is no loss of generality in assuming that $f$ satisfies the hypotheses of Lemma 3. For otherwise we can replace $f$ by a function $W$ which we construct as follows. Let $\gamma$ be a crosscut of $D$ meeting $J$ at its endpoints, and let $G$ be the subdomain of $D$ bounded by $J \cup \gamma$. Suppose without loss of generality that $0 \in G$, and let $\psi: D \rightarrow G$ be a conformal map satisfying $\psi(0)=0$. Then the function defined by $W=\left(1 / \psi^{\prime}(0)\right) f \circ \psi$ satisfies the hypotheses of $f$ in Lemma 3. Since the desired result is intrinsic to $f(D)$ and not to $W$, our claim follows at once.

Hence, suppose that $f$ is exactly as in Lemma 3. Also, suppose that $h^{\prime}(z) \neq 0$ for all $z \in J$; otherwise we take a subinterval of $J$ with this condition. Let $\zeta_{1}, \zeta_{2}$ be a pair of distinct points of $J$, and $I$ the subinterval of $J$ with endpoints $\zeta_{1}$ and $\zeta_{2}$. Also let $l_{1}, l_{2}$ be the radii of $D$ ending at $\zeta_{1}, \zeta_{2}$ respectively, $S$ the closed sector bounded 
by $I \cup l_{1} \cup l_{2}, \Omega=f(S), L_{1}=f\left(l_{1}\right)$, and $L_{2}=f\left(l_{2}\right)$. Then it follows directly that $\Omega$ is the simply connected subdomain of $f(D)$ bounded only by $L_{1} \cup L_{2}$.

First, we observe that the inner angle of $\Omega$ at $w_{0}$ exists and has size

$$
\begin{aligned}
\left|\arg \frac{f_{r}\left(\zeta_{1}\right)}{f_{r}\left(\zeta_{2}\right)}\right| & =\left|\arg \frac{\zeta_{1} h^{\prime}\left(\zeta_{1}\right)+\overline{\zeta_{1} g^{\prime}\left(\zeta_{1}\right)}}{\zeta_{2} h^{\prime}\left(\zeta_{2}\right)+\overline{\zeta_{2} g^{\prime}\left(\zeta_{2}\right)}}\right| \\
& =\left|\arg \frac{\zeta_{1} h^{\prime}\left(\zeta_{1}\right)}{\zeta_{2} h^{\prime}\left(\zeta_{2}\right)}\right|
\end{aligned}
$$

because of Lemma 3.c.

Now suppose that $f(D)$ does not satisfy the wedge condition at $w_{0}$. Then it is immediate in view of the above that

$$
\left|\arg \frac{f_{r}\left(\zeta_{1}\right)}{f_{r}\left(\zeta_{2}\right)}\right|=0
$$

for all $\zeta_{1}, \zeta_{2} \in J$; or equivalently,

$$
z h^{\prime}(z)=\left|z h^{\prime}(z)\right| e^{i \eta}, \quad z \in \operatorname{Int}(J),
$$

where $\eta$ is a constant. Then by Lemma 3 we also have

$$
z g^{\prime}(z)=\left|z h^{\prime}(z)\right| e^{-i \eta}, \quad z \in \operatorname{Int}(J),
$$

Hence

$$
\frac{h^{\prime}}{g^{\prime}}(z)=e^{2 i \eta}, \quad z \in \operatorname{Int}(J),
$$

and $h^{\prime} / g^{\prime}$ is a constant function in $D$ by the identity theorem, and $\left|h^{\prime}\right|=\left|g^{\prime}\right|$ in $D$. This contradicts that $f \in S_{H}$. Therefore, our assumption is false and $f(D)$ satisfies the wedge condition at $w_{0}$.

REMARK. The conclusion of theorem is best possible. In other words, $P$ may not be a prime-end of the first kind, that is the impression of $P, I(P)$, is non-degenerate, as shown by the following example.

EXAMPLE 1. Let

$$
K_{0}(z)=\operatorname{Re} \frac{z+\frac{1}{3} z^{3}}{(1-z)^{3}}+i \operatorname{Im} \frac{z}{(1-z)^{2}} .
$$

It was shown in [3] that $K_{0} \in S_{H}, K_{0}$ maps $D$ to the complement of the ray on the negative real-axis from $-1 / 6$ to $\infty$ and $K_{0}(\zeta)=-1 / 6$ for all $\zeta \in \partial D \backslash\{1\}$. Let $\alpha$ be the circular arc in $D$ with endpoints 1 , $i$ and perpendicular to $\partial D$ (see Figure 1). Let $\beta=K_{0}(\alpha), G$ the lens domain between $\partial D$ and $\alpha$ and $H=K_{0}(G)$. Then $H$ is the domain in the upper half-plane bounded by $\beta$ and $[-\infty,-1 / 6]$. For $n=1,2, \ldots$ 

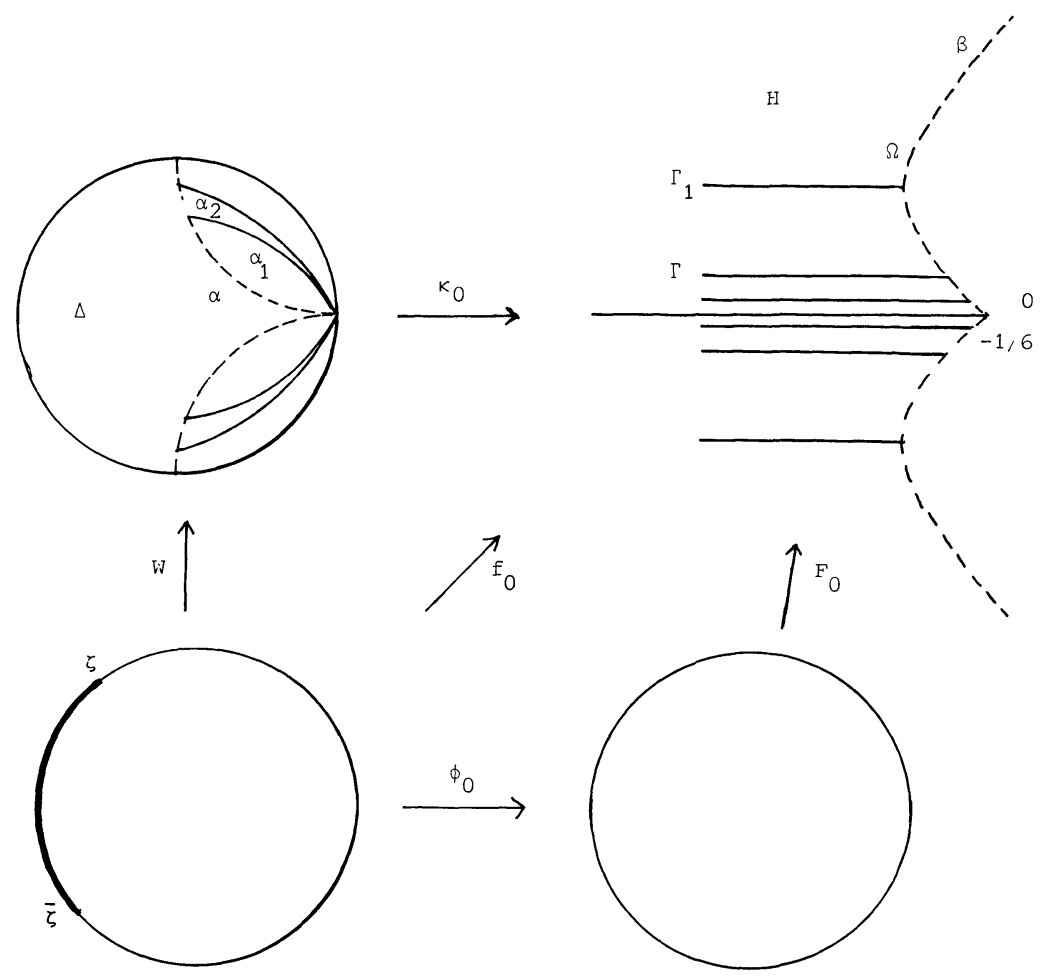

FIGURE 1

we let $\Gamma_{n}$ to be the horizontal slit in $\bar{H}$ ending at $\beta$ and satisfying $\operatorname{Im} w=\frac{1}{n}$ for $w \in \Gamma_{n}$ (see Figure 1). Then the $\operatorname{arcs} K_{0}^{-1}\left(\Gamma_{n}\right)=\alpha_{n}$ lie in $G$ and converge to $\partial D$ as shown. Let $\bar{A}$ denote the reflection of $A$ about the real axis. Now we let

$$
\begin{aligned}
& W: D \rightarrow D \backslash \bigcup_{j=1}^{\infty}\left(\alpha_{n} \cup \bar{\alpha}_{n}\right)=\Delta \\
& F_{0}: D \rightarrow K_{0}(D) \backslash \bigcup_{n=1}^{\infty}\left(\Gamma_{n} \cup \bar{\Gamma}_{n}\right)=\Omega
\end{aligned}
$$

be the two conformal maps satisfying $W(0)=F_{0}(0)=0$ and $W^{\prime}(0)$, $F^{\prime}(0)>0$ and define

$$
\begin{aligned}
f_{0} & =K_{0} \circ W, \\
\phi_{0} & =F_{0}^{-1} \circ f_{0} .
\end{aligned}
$$

Observe that $\Delta$ has two accessible prime-ends $P_{1}, P_{2}$ of non-degenerate impressions with accessible points $i,-i$ and $\Omega$ has one accessible prime end $P$ with impression $(-\infty,-1 / 6]$, accessibility point $-1 / 6$ 
and which satisfy the wedge condition. Note that there exist symmetric points $\zeta, \bar{\zeta} \in \partial D(\zeta \neq-1,1)$ corresponding, under $W$, to $P_{1}$ and $P_{2}$ respectively, and that -1 corresponds, under $F_{0}$, to $P$. Then $f_{0}$ extends continuously to the circular arc ending at $\zeta$ and $\bar{\zeta}$ and containing $-1, f_{0} \equiv-1 / 6$ there, and $f_{0}(D)$ satisfies the wedge condition at $P$.

We finally prove two corollaries which extend a result for normal functions [8, p. 267].

Corollary 2. Let $f=u+v \in S_{H}$ satisfy $u, v \in h^{p}, p>1$, and $\alpha: z(t), 0 \leq t \leq 1$, be a Jordan arc in $D$ with $|z(t)| \rightarrow 1(t \rightarrow 1)$. If $f(z(t)) \rightarrow w_{0}(t \rightarrow 1)$ and

$$
\lim _{t \rightarrow 1^{-}} \arg \left(f(z(t))-w_{0}\right)
$$

exists, then $z(t) \rightarrow \zeta(t \rightarrow 1)$ for some $\zeta \in \partial D$ so that $\alpha$ is an asymptotic path.

For the definition of an asymptotic path see [8, p. 267].

Proof. Suppose that the set of accumulation points of $\alpha$ on $\partial D$ form a nondegenerate arc $I$. Then $f(\zeta)=w_{0}$ for every $\zeta \in I$ where $f(\zeta)$ exists. Because radial limits exist a.e. and $u, v \in h^{p}, p>1, f$ extends continuously to a constant function in $\operatorname{Int}(I)$. Using this, we conclude from the proof of Theorem 3 that for distinct $\zeta_{1}, \zeta_{2} \in \operatorname{Int}(I)$ the images $l_{1}, l_{2}$, under $f$, of the radii ending at $\zeta_{1}, \zeta_{2}$, respectively, are Jordan half-intervals in $f(D)$, both ending at $w_{0}$ and make, in $f(D)$, an angle of positive size at $w_{0}$. This contradicts (10), since each of the arcs $l_{1}$ and $l_{2}$ meets $f(\alpha)$ in a sequence converging to $w_{0}$, and the proof is complete.

REMARK. Condition (10) of Corollary 2 is essential. For example:

EXAMPLE 2. Let $f$ be the function defined by

$$
f\left(e^{i \theta}\right)= \begin{cases}1, & 0 \leq \theta<2 \pi / 3 \\ i, & 2 \pi / 3 \leq \theta<4 \pi / 3 \\ -i, & 4 \pi / 3 \leq \theta<2 \pi\end{cases}
$$

and

$$
f(z)=\frac{1}{2 \pi} \int_{0}^{2 \pi}\left(\operatorname{Re} \frac{e^{i \theta}+z}{e^{i \theta}-z}\right) f\left(e^{i \theta}\right) d \theta .
$$

Then $f$ is a univalent harmonic map and $f(D)$ is a triangle (see [3]). Let $\gamma$ be a curve in $D$ converging to a non-degenerate arc subset to the arc $\left\{e^{i \theta}: 0 \leq \theta \leq \pi / 2\right\}$. Then $f(\gamma)$ converges to 1 . 
Corollary 3. Let $f$ be as in Corollary 2, $w_{0}$ an accessible point of $f(D)$, and $\alpha_{j}: z_{j}(t), 0 \leq t<1(j=1,2)$ be Jordan arcs in $D$ with $\left|z_{j}(t)\right| \rightarrow 1(t \rightarrow 1)$. If $f\left(z_{j}(t)\right) \rightarrow w_{0}(t \rightarrow 1)$,

$$
\lim _{t \rightarrow 1^{-}} \arg \left(f\left(z_{j}(t)\right)-w_{0}\right)=\Theta \quad(j=1,2)
$$

and the zero cusp at $w_{0}$ with sides $f\left(\alpha_{j}\right)$ lies in $f(D)$, then $z_{j}(t) \rightarrow \zeta$ $(t \rightarrow 1 ; j=1,2)$ for some $\zeta \in \partial D$.

Proof. It follows at once from Corollary 2 that each $\alpha_{j}$ is an asymptotic path with $z_{j}(t) \rightarrow \zeta_{j}(t \rightarrow 1)$. If $\zeta_{1} \neq \zeta_{2}$, then there exists a Jordan arc $\Gamma: z(t), 0 \leq t<1$, in $D$ such that for some positive sequence $t_{n} \rightarrow 1^{-} \Gamma$ meets $\alpha_{j}$ at $z_{j}\left(t_{n}\right)(j=1,2)$ for all $n, f(z(t)) \rightarrow w_{0}$ $(t \rightarrow 1)$, and

$$
\lim _{t \rightarrow 1} \arg \left(f(z(t))-w_{0}\right)=\Theta .
$$

By Corollary $2 \Gamma$ is an asymptotic path, a contradiction. This completes the proof.

REMARK. Condition (11) is essential. For example, let $f$ be as in Example 2 and $\alpha_{1}, \alpha_{2}$ be two disjoint curves terminating at $e^{i \pi / 4}$ and $i$. Then $f\left(\alpha_{1}\right), f\left(\alpha_{2}\right)$ terminate at 1 with a positive angle between them.

4. Correspondence under $f$ between $D$ and the prime-ends of $f(D)$. Throughout this section, we let

$$
\begin{aligned}
& E=\{\zeta \in \partial D: C(\phi, \zeta) \text { is not a singleton }\}, \text { and } \\
& G=\left\{\lambda \in \partial D: C\left(\phi^{-1}, \lambda\right) \text { is not a singleton }\right\}
\end{aligned}
$$

where $\phi$ is as given by $(8)$ and $C(\phi, \zeta)$ is the cluster set of $\phi$ at $\zeta$. For the definition of a cluster set, see [1, p. 66] or [8, p. 276]. In addition, let $C_{R}(\phi, \zeta)$ and $C_{L}(\phi, \zeta)$ denote the right and left cluster sets of $\phi$ at $\zeta[1$, p. 83].

We next state Theorem 4, whose proof is a consequence of Theorem 3 and the forthcoming Lemma 5.

THEOREM 4. Let $\lambda \in G$. Then the prime-end $P$ of $f(D)$ corresponding to $\lambda$, under $F$, is accessible and satisfies the wedge condition at $P$.

The next lemma is purely topological and is true for all automorphisms of $D$ with radial limits almost everywhere. 
LEMMA 4. Let $\zeta, \zeta^{\prime} \in \partial D$.

(a) If $C(\phi, \zeta)=\partial D$, then $\phi$ extends continuously to a constant $\lambda$, $|\lambda|=1$, on $\partial D \backslash \zeta$, and $\zeta$ is unique.

(b) If $C(\phi, \zeta), C\left(\phi, \zeta^{\prime}\right)$ are not $\partial D$, then $\operatorname{Int} C(\phi, \zeta) \cap C\left(\phi, \zeta^{\prime}\right)=$ $C(\phi, \zeta) \cap \operatorname{Int} C\left(\phi, \zeta^{\prime}\right)=\phi$.

(c) If $C(\phi, \zeta) \cap C\left(\phi, \zeta^{\prime}\right)=\lambda$, $|\lambda|=1$, then $\phi$ extends continuously to an open circular arc between $\zeta$ and $\zeta^{\prime}$, and $\phi \equiv \lambda$ there.

Proof. (a) Let $\gamma$ be a union of two radii of $D$ so that $\zeta$ and $\zeta^{\prime}$ are on different sides of $\gamma$ and $\phi$ has radial limits along these radii. Since $C(\phi, \zeta)=\partial D, \phi(\gamma)$ is a loop that meets $\partial D$ at a unique point $\lambda$. We infer at once that $C\left(\phi, \zeta^{\prime}\right)=\lambda$ for all $\zeta^{\prime} \neq \zeta$, and $\zeta$ is unique. This proves (a).

(b) Choose $\zeta_{1}, \zeta_{2} \in \partial D$, different from $\zeta, \zeta^{\prime}$, so that the radial limits $\hat{\phi}\left(\zeta_{1}\right), \hat{\phi}\left(\zeta_{2}\right)$ exist and the crosscup $\gamma$, made up of the radii terminating at $\zeta_{1}$ and $\zeta_{2}$, separates $\zeta$ and $\zeta^{\prime}$ in $D$. Then the crosscut, $\phi(\gamma)$ of $D$, separates $D$ into two simply connected domains such that one contains $C(\phi, \zeta)$ on its boundary and the other $C\left(\phi, \zeta^{\prime}\right)$. This proves (b).

(c) If $C(\phi, \zeta)=\partial D$ for some $\alpha \in \partial D$, then we are done. Suppose otherwise and let $I, I^{\prime}$ be the subarcs of $\partial D$ determined by $\zeta, \zeta^{\prime}$. If for every $\zeta_{1} \in \operatorname{Int}(I), \phi(\alpha)$ exists and $\phi\left(\zeta_{1}\right)=\lambda$, then (c) follows, otherwise choose $\zeta_{1} \in \operatorname{Int}(I), \zeta_{2} \in \operatorname{Int}\left(I^{\prime}\right)$ such that $\phi\left(\zeta_{1}\right), \phi\left(\zeta_{2}\right)$ exists and $\phi\left(\zeta_{1}\right) \neq \lambda$. Then an argument similar to that of (b) yields (c) and the proof is complete.

REMARK. (1) $K_{0}$ indicates that (a) is possible.

(2) It follows from Lemma 4, that the set $E$ (see [12]) is at most countable.

LEMMA 5. Let $\zeta, \zeta^{\prime} \in \partial D$.

(a) If $C(\phi, \zeta)=\partial D$, then $f$ extends continuously to $\partial D \backslash \zeta$ and $f$ is constant there.

(b) If $C(\phi, \zeta) \cap C\left(\phi, \zeta^{\prime}\right)=\lambda$, $|\lambda|=1$, then $f$ extends continuously to an open circular arc between $\zeta$ and $\zeta^{\prime}$, and $f \equiv F(\lambda)$ there.

Proof. (a) By Lemma $4 \phi$ extends continuously to some constant, $\lambda,|\lambda|=1$ on $\partial D \backslash \zeta$. Let $\zeta_{1}, \zeta_{2} \in \partial D \backslash \zeta$ be chosen such that $f\left(\zeta_{1}\right)$, $f\left(\zeta_{2}\right)$ exist. If $\gamma_{1}, \gamma_{2}$ are the images, under $\phi$, of the radii ending at $\zeta_{1}, \zeta_{2}$, respectively, then $\gamma_{1}, \gamma_{2}$ are asymptotic paths of $F$ both ending at $\lambda$. Since $F$ is univalent, $F$ is normal (see [8, pp. 262-263]), and 
$F\left(\zeta_{1}\right)=F\left(\zeta_{2}\right)$ (see [8, p. 267]). Therefore, $f\left(\zeta_{1}\right)=f\left(\zeta_{2}\right)$. Since $\zeta_{1}$, $\zeta_{2}$ are arbitrary, the desired result follows.

The truth of (b) follows in the same manner. This completes the proof.

As an immediate consequence of Theorem 4 we have

Corollary 4. Suppose that $f(D)$ does not satisfy the wedge condition at the prime-end $P$ corresponding to $\lambda \in \partial D$; then there exists a unique $\zeta \in \partial D$ such that $\lambda \in C(\phi, \zeta)$.

In what follows, we assume that $f=u+i v$ satisfies $u, v \in h^{p}, p>1$.

Let $[a, b], a \neq b \in \mathbb{C} \cup\{\infty\}$, denote the line segment from $a$ to $b$. We call $[a, b]$ a side of a domain $H$ if no point of the interior, $(a, b)$, of $[a, b]$ is a point of accumulation of $\partial H \backslash[a, b]$ and $H$ lies on the left of $[a, b]$. For $\lambda_{1}, \lambda_{2} \in \partial D$, let $\overline{\lambda_{1} \lambda_{2}}$ be the circular arc described by going positively from $\lambda_{1}$ to $\lambda_{2}$.

Suppose that $f$ is defined on an open subarc $\left\{e^{i \theta}: \theta_{0}-\delta<\theta<\right.$ $\left.\theta_{0}+\delta\right\}$ of $\partial D$. We say that $f$ is constant on the right (left) of $e^{i \theta_{0}}$ if $f\left(e^{i \theta}\right)$ is constant for all $\theta, \theta_{0}-\delta<\theta<\theta_{0}\left(\theta_{0}<\theta<\theta_{0}+\delta\right)$.

The following theorem describes the cluster set of $f$ at a point in $E$.

THEOREM 5. Let $f=u+i v, u, v \in h^{p}, p>1, \zeta_{0}=e^{i \theta_{0}} \in E$ and $C\left(\phi, \zeta_{0}\right)=\overline{\lambda_{1} \lambda_{2}}$. Then

$$
C\left(f, \zeta_{0}\right)=I_{1} \cup[a, b] \cup I_{2},
$$

where $[a, b]$ is a side of $\partial f(D)$, and $I_{1}=a\left(I_{2}=b\right)$ if $f$ is constant on the right (left) of $\zeta_{0}$, otherwise $I_{1}=I\left(P_{1}\right)\left(I_{2}=I\left(P_{2}\right)\right)$, where $P_{1}$ are $P_{2}$ are the prime ends of $f(D)$ corresponding to $\lambda_{1}$ and $\lambda_{2}$, respectively. In this case, $P_{1}\left(P_{2}\right)$ is accessible at a $(b)$, and $I\left(P_{1}\right)\left(I\left(P_{2}\right)\right)$ has at most one continuum of subsidiary points given by $C_{R}\left(F, \lambda_{1}\right)\left(C_{L}\left(F, \lambda_{2}\right)\right)$.

Proof. We show first that $\phi^{-1}$ has radial limits a.e. on $C\left(\phi, \zeta_{0}\right)$. Choose $\lambda_{0} \in \operatorname{Int} C\left(\phi, \zeta_{0}\right)$ so that $F\left(\lambda_{0}\right)$ exists, and let $\gamma_{0}$ be the radius ending at $\lambda_{0}$, and $\alpha_{0}=\phi^{-1}\left(\gamma_{0}\right)$. We claim that $\alpha_{0}$ is a Jordan halfinterval ending at $\zeta_{0}$. Let $J$ denote the interval of accumulation points of $\alpha_{0}$ on $\partial D$. Since $u, v \in h^{p} ; p>1, J$ is a proper subinterval of $\partial D$; otherwise $f$ is identically $F\left(\lambda_{0}\right)$, a contradiction. Pick $\zeta \in(\operatorname{Int}(J)) \backslash \zeta_{0}$, and $\zeta^{\prime} \in J$ so that $\phi\left(\zeta^{\prime}\right)$ exists and $\phi\left(\zeta^{\prime}\right) \neq \lambda_{0}$, and let $\alpha, \alpha^{\prime}$ be the radii ending at $\zeta, \zeta^{\prime}$, respectively. Then $\phi(\alpha) \cup \phi\left(\alpha^{\prime}\right)$ is a crosscut of 
$D$ dividing $D$ into two simply connected domains the boundary of each of which contains a subarc of $C\left(\phi, \zeta_{0}\right)$ not shared by the other, a contradiction.

Now we choose $\lambda_{1}^{\prime}, \lambda_{2}^{\prime} \in C\left(\phi, \zeta_{0}\right)$ so that $\widehat{\lambda_{1}^{\prime} \lambda_{2}^{\prime}}$ lies in $\widehat{\lambda_{1} \lambda_{2}}$, and $F\left(\lambda_{1}^{\prime}\right)$, $F\left(\lambda_{2}^{\prime}\right)$ exist. For $j=1,2$, let $\gamma_{j}$ be the radius ending at $\lambda_{j}^{\prime}, \beta_{j}=F\left(\gamma_{j}\right)$ and $\alpha_{j}=\phi^{-1}\left(\gamma_{j}\right)$. Then $\alpha_{1} \cup \alpha_{2}$ is a loop which, except for $\zeta_{0}$, lies in $D$, and bounds a simply connected subdomain, $G$, of $D$. If $W: D \rightarrow G$ is a conformal map satisfying $W(1)=\zeta_{0}$, and $H=f \circ W$, then $H$ is a harmonic univalent map which equals $F\left(\lambda_{1}^{\prime}\right)$ on the right of 1 and $F\left(\lambda_{2}^{\prime}\right)$ on the left, and $\operatorname{Re} H, \operatorname{Im} H \in h^{p}, p>1$, by Littlewood's subordination theorem [4, pp. 10-11]. Call $\Omega$ the domain bounded by $\beta_{1} \cup \beta_{2} \cup\left[F\left(\lambda_{1}^{\prime}\right), F\left(\lambda_{2}^{\prime}\right)\right]$, and $S$ the sector bounded by $\gamma_{1} \cup \gamma_{2} \cup \overline{\lambda_{1}^{\prime} \lambda_{2}^{\prime}}$. Then $C(H, 1)=\left[F\left(\lambda_{1}^{\prime}\right), F\left(\lambda_{2}^{\prime}\right)\right]$ by a theorem of Schwarz [9, p. 131], the cluster set of $f$ at $\zeta$ within $G$ is $\left[F\left(\lambda_{1}^{\prime}\right), F\left(\lambda_{2}^{\prime}\right)\right]$, and $F$ extends to a homeomorphism between $\bar{S}$ and $\bar{\Omega}$ mapping $\widehat{\lambda_{1}^{\prime} \lambda_{2}^{\prime}}$ to $\left[F\left(\lambda_{1}^{\prime}\right), F\left(\lambda_{2}^{\prime}\right)\right]$. Letting $\lambda_{1}^{\prime} \rightarrow \lambda_{1}$ and $\lambda_{2}^{\prime} \rightarrow \lambda_{2}$, then $F\left(\lambda_{1}^{\prime}\right) \rightarrow a$ and $F\left(\lambda_{2}^{\prime}\right) \rightarrow b$, where $a, b \in \mathbb{C} \cup\{\infty\}$, and $C\left(f, \zeta_{0}\right)$ contains $[a, b]$ which obviously is a side of $f(D)$.

Now suppose that $f$ is constant on the left (right) of $\zeta_{0}$, then by replacing $\alpha_{1}\left(\alpha_{2}\right)$ by some circular arc $\left\{e^{i \theta}: \theta_{0}-\delta \leq \theta<\theta_{0}\right\}\left(\left\{e^{i \theta}: \theta_{0}<\right.\right.$ $\left.\left.\theta \leq \theta_{0}+\delta\right\}\right)$ and carrying the same argument above, we infer that $I_{1}=a\left(I_{2}=b\right)$.

Suppose to the contrary that $f$ is not constant either on the right or on the left of $\zeta_{0}$, then because $u, v \in h^{p}, p>1$, there exist $\theta_{n} \theta_{n}^{\prime}, \theta_{n} \rightarrow$ $\theta_{0}^{-}, \theta_{n}^{\prime} \rightarrow \theta_{0}^{+}$and $r_{n} \rightarrow 1^{-}$such that if, for $n=1,2, \ldots$,

$$
Q_{n}=\left(e^{i \theta_{n}}, r_{n} e^{i \theta_{n}}\right] \cup\left\{r_{n} e^{i t}: \theta_{n} \leq t \leq \theta_{n}^{\prime}\right\} \cup\left[r_{n} e^{i \theta_{n}^{\prime}}, e^{i \theta_{n}^{\prime}}\right),
$$

then $\phi\left(Q_{n}\right)$ is a crosscut of $D$ (see [8, p. 267]) which meets $\widetilde{\lambda_{1} \lambda_{2}}$ nowhere, and $\phi\left(Q_{n}\right) \rightarrow \widehat{\lambda_{1} \lambda_{2}}(n \rightarrow \infty)$. Hence $I_{1}=I\left(P_{1}\right)$ and $I_{2}=$ $I\left(P_{2}\right)$. It is also immediate that $P_{1}$ and $P_{2}$ are accessible at $a$ and $b$, respectively, since $F$ extends conformally to $\operatorname{Int}\left(\widetilde{\lambda_{1} \lambda_{2}}\right)$ and maps $\operatorname{Int}\left(\widetilde{\lambda_{1} \lambda_{2}}\right)$ to $(a, b)$. Therefore the radial limits $F\left(\lambda_{1}\right), F\left(\lambda_{2}\right)$ exist and they are $a, b$, respectively. Consequently, by Lindelof's theorem [1, p. 72] $C_{L}\left(F, \lambda_{1}\right)=a$ and $C_{R}\left(F, \lambda_{2}\right)=b$, which forces each of $P_{1}$ and $P_{2}$ to have at most one continuum of subsidiary points given by $C_{R}\left(F, \lambda_{1}\right)$ and $C_{L}\left(F, \lambda_{2}\right)$, respectively, (see [1, pp. 188-189]). This completes the proof. 


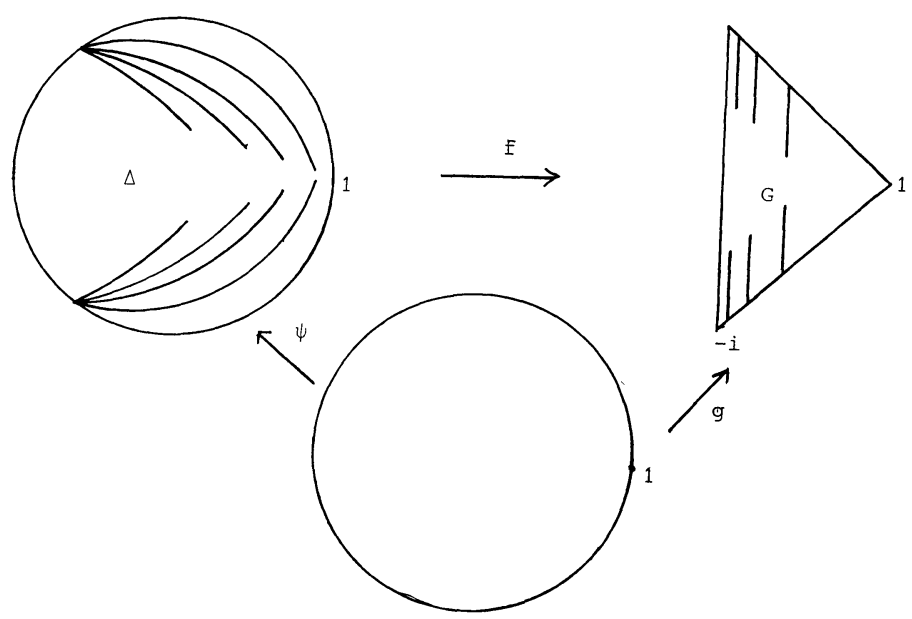

FIGURE 2

EXAMPLE 3. Let $f$ be the function defined in Example 2. For $n=2,3, \ldots$, let $\Gamma_{n}: w(t)=\frac{1}{n}+i t\left(\frac{1}{2}\left(1-\frac{1}{n}\right) \leq t \leq 1-\frac{1}{n}\right)$ and $\bar{\Gamma}_{n}$ be the reflection of $\Gamma_{n}$ about the real axis. Then $G=f(D) \backslash\left(\bigcup_{n=2}^{\infty}\left(\Gamma_{n} \cup \bar{\Gamma}_{n}\right)\right)$ is the simply connected domain shown in Figure 2. Let $\gamma_{n}=f^{-1}\left(\Gamma_{n}\right)$ and $\bar{\gamma}_{n}$ be the reflection of $\gamma_{n}$ about the real axis, for all $n$. Because of the symmetry $\overline{f(z)}=f(\bar{z})$, it follows that $\bar{\gamma}_{n}=f^{-1}\left(\bar{\Gamma}_{n}\right)$. It is immediate that each of the arcs $\gamma_{n}$ and $\bar{\gamma}_{n}$ is an asymptotic path, under $f$, in $D$ meeting $\partial D$ at $e^{i 2 \pi / 3}$ and $e^{i 4 \pi / 3}$, respectively, such that the sequences $\left(\gamma_{n}\right)$ and $\left(\bar{\gamma}_{n}\right)$ converge uniformly to the circular arcs $\left\{e^{i \theta}: 0 \leq \theta \leq 2 \pi / 3\right\}$ and $\left\{e^{i \theta}: 4 \pi / 3 \leq \theta \leq 2 \pi\right\}$, respectively. If

$$
\Delta=D \backslash\left(\bigcup_{n=2}^{\infty}\left(\gamma_{n} \cup \bar{\gamma}_{n}\right)\right),
$$

then $\Delta$ is a simply connected domain symmetric about the real axis and has a prime end, $P$, accessible at 1 , with impression the $\operatorname{arc}\left\{e^{i \theta}:-\right.$ $2 \pi / 3 \leq \theta \leq 2 \pi / 3\}$, see Figure 2. So if $\psi: D \rightarrow \Delta$ is the Riemann mapping satisfying $\psi(0)=0$ and $\psi^{\prime}(0)>0$, then 1 corresponds, in the Carathéodory sense, to $P$ under $\psi$. Furthermore, it can be easily verified that the harmonic univalent map $g=f \circ \psi$ has a nonempty set $E$ such that $1 \in E$ and $C(g, 1)$ satisfies the conclusion of Theorem 5 .

The next two corollaries follow from Theorem 5 .

COROLlary 5. Under the assumptions of Theorem 5 , if $f(D)$ has no sides, then $E=\varnothing$. 
COROLlaRY 6. Under the assumptions of Theorem 5, if $P$ is a prime end of $f(D)$ which is either non-accessible, or accessible but has two subsidairy continua without the wedge condition, then there exists a unique $\zeta \in \partial D$, with $\zeta \in \partial D \backslash E$ such that $C(\phi, \zeta)=\lambda$, where $\lambda$ is the corresponding point to $P$ under $F$.

In the final part of this section, we give conclusive statements of correspondence between various points of $\partial D$ and the prime-ends of $f(D)$, where $f$ is assumed univalent and in $h^{p}, p>1$. First we need the following definition.

Definition 3. Let $A$ and $B$ be subsets of $\partial D$ ( $B$ corresponds to a set of prime ends of $f(D)$ under $F)$. Then we say

(a) $A$ corresponds to $B$ if $C(\phi, \zeta) \subset B$ and $C\left(\phi, \zeta^{\prime}\right) \subset \partial D \backslash B$ for all $\zeta \in A$ and $\zeta^{\prime} \in \partial D \backslash A$.

(b) $B$ corresponds to $A$ if $C\left(\phi^{-1}, \zeta\right) \subset A$ and $C\left(\phi^{-1}, \zeta^{\prime}\right) \subset \partial D \backslash A$ for all $\zeta \in B$ and $\zeta^{\prime} \in \partial D \backslash B$.

(c) $A$ and $B$ correspond to each other if (a) and (b).

(d) There is one to one correspondence between $A$ and $B$ if every point of $A$ corresponds to a single point of $B$ and conversely.

In accordance with this definition, if $\mathscr{A}$ is the set of all $\zeta \in \partial D \backslash E$ where $f$ is constant neither on the left nor the right of $\zeta$, and $\mathscr{B}=$ $\{\hat{\phi}(\zeta): \zeta \in A\}$, then Theorem 3 and an argument of the proof of Theorem 5 yield the following:

THEOREM 6. There is a one-to-one correspondence between $\mathscr{A}$ and $\mathscr{B}$.

In view of this result, special attention need be given to the case when $f$ is constant on some non-degenerate open subinterval of $\partial D$. Let $J=\widehat{\zeta_{1} \zeta_{2}}$ be such a subinterval. Since Schwarz's Theorem [9, p. 131] offers an exact description of $f$ at $\zeta_{1}\left(\zeta_{2}\right)$ if $f$ is also constant on the right of $\zeta_{1}$ (left of $\zeta_{2}$ ), we restrict ourselves to the complementary case when $f$ is constant neither on the right of $\zeta_{1}$ nor the left of $\zeta_{2}$.

THEOREM 7. Suppose that $f(\zeta) \equiv w$ for all $\zeta \in \operatorname{Int} \overline{\zeta_{1} \zeta_{2}}$ and $f$ is constant neither on the right of $\zeta_{1}$ nor the left of $\zeta_{2}$. Then the following is true:

(a) If $\zeta_{1}, \zeta_{2} \in \partial D \backslash E$, then there is a prime end, $\lambda$, of $f(D)$ satisfying the wedge condition at $w_{0}$ such that $\widehat{\zeta}_{1} \zeta_{2}$ and $\lambda$ correspond to each other with $C\left(\phi, \zeta_{1}\right)=C_{R}(F, \lambda)$ and $C\left(\phi, \zeta_{2}\right)=C_{L}(F, \lambda)$ (see Figure 3.a). 


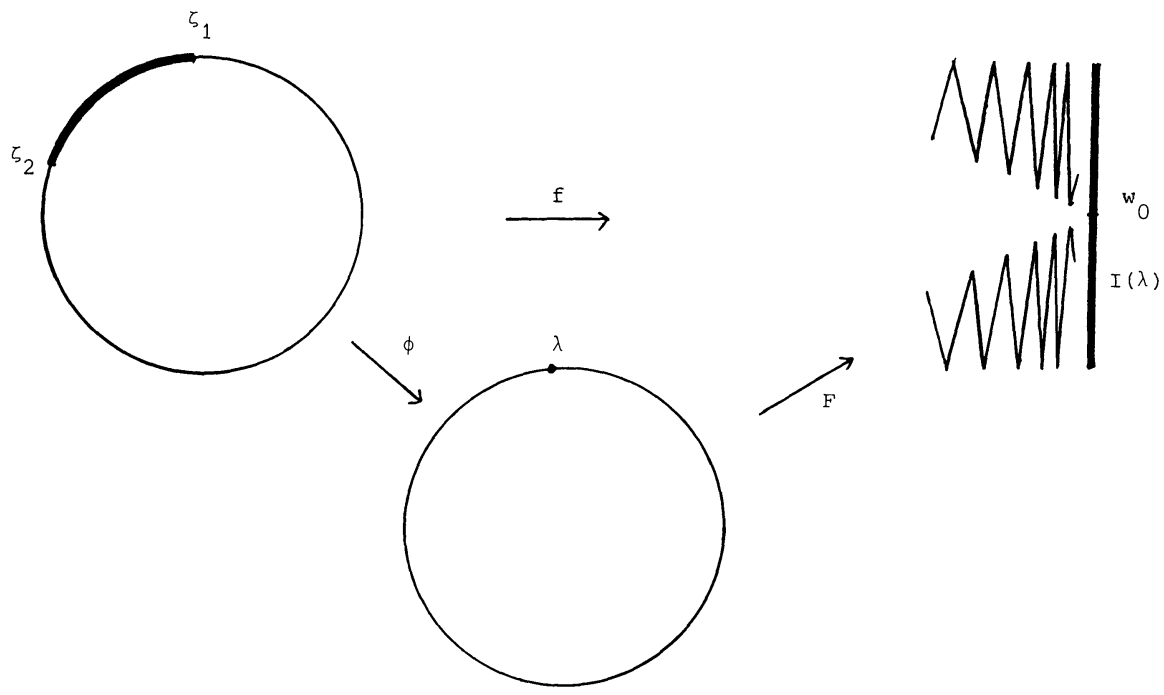

Figure 3.a

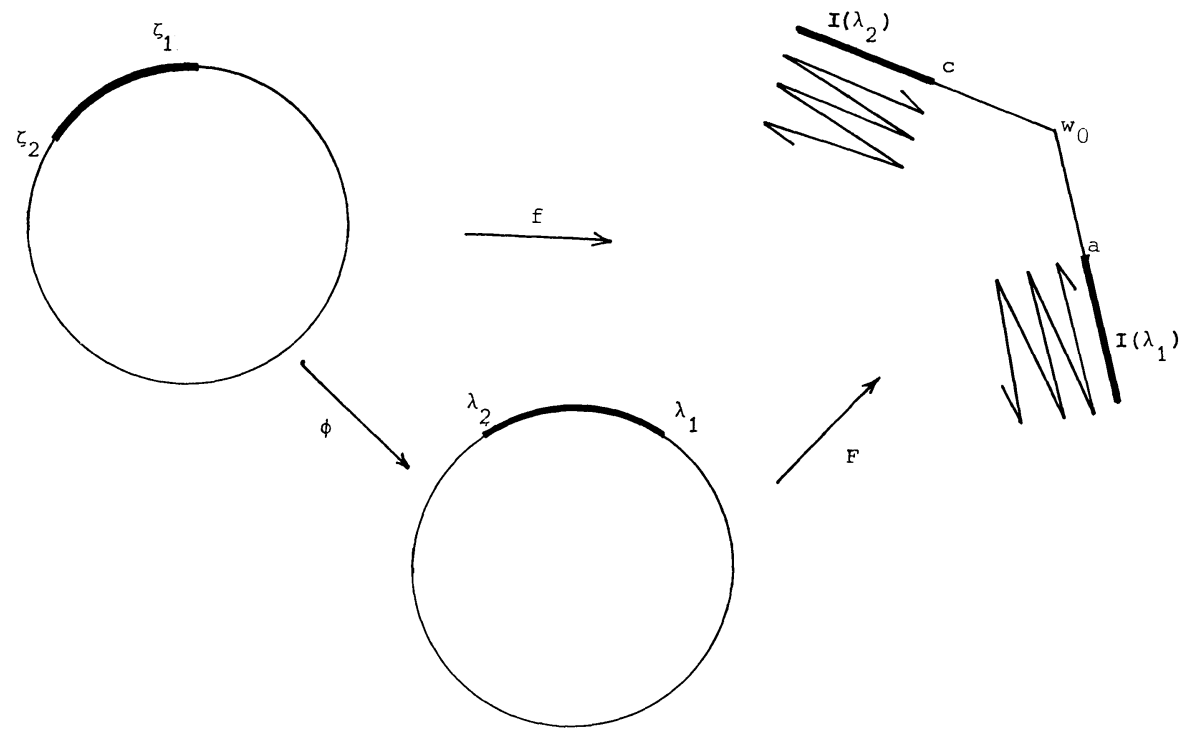

Figure 3.b

(b) If $\zeta_{1}, \zeta_{2} \in E$, then there is a non-degenerate interval $\widehat{\lambda_{1} \lambda_{2}}$ of prime ends of $f(D)$ such that $\overline{\zeta_{1} \zeta_{2}}$ and $\overline{\lambda_{1} \lambda_{2}}$ correspond to each other. Both of $\lambda_{1}$ and $\lambda_{2}$ are (prime ends) accessible, say at $a$ and c, respectively, and the impressions of all the prime ends in $\lambda_{1} \hat{\lambda}_{2}$ is $I\left(\lambda_{1}\right) \cup\left[a, w_{0}\right] \cup\left[w_{0}, c\right] \cup$ $I\left(\lambda_{2}\right)$. (See Figure 3.b) 


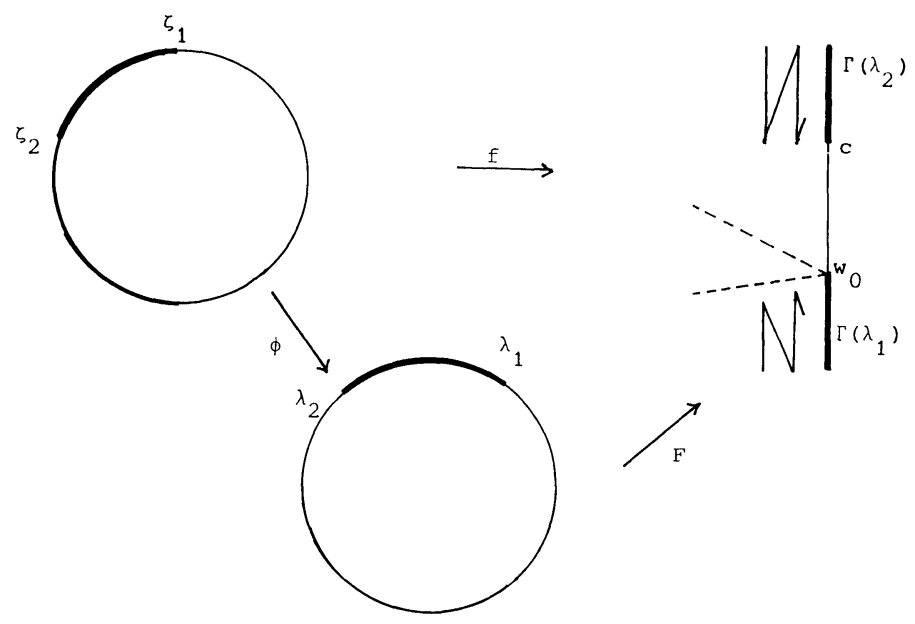

FIGURE 3.c

(c) If $\zeta_{1} \in \partial D \backslash E$ and $\zeta_{2} \in E$, then there is a non-degenerate interval $\overline{\lambda_{1} \lambda_{2}}$ of prime ends of $f(D)$ such that $\overline{\zeta_{1} \zeta_{2}}$ and $\overline{\lambda_{1} \lambda_{2}}$ correspond to each other. Both of $\lambda_{1}$ and $\lambda_{2}$ are (prime ends) accessible, say at $w_{0}$ and $c$, respectively, and the former satisfy the wedge condition. Furthermore, the impressions on all the prime ends is $I\left(\lambda_{1}\right) \cup\left[w_{0}, c\right] \cup I\left(\lambda_{2}\right)$ (see Figure 3.c).

The proof of this theorem follows immediately from Theorems 3, 4 and 5 and the fact that $f \in h^{p}, p>1$.

REMARK. Wherever and throughout the paper, the assumption $f \in$ $h^{p}, p>1$ or equivalently $u, v \in h^{p}, p>1$ can be replaced by the property that sets of positive measure on $\partial D$ are sets of uniqueness for $f$ or $u, v$.

\section{REFERENCES}

[1] E. Collingwood and A. Lohwater, The Theory of Cluster Sets, Cambridge University Press, 56, (1966).

[2] J. G. Clunie and T. H. MacGregor, Radial growth of the derivative of the univalent function, Comment. Math. Helv., 59, No. 3, (1984), 362-375.

[3] J. G. Clunie and T. Sheil-Small, Harmonic univalent functions, Ann. Acad. Sci. Fenn. Ser. A.I., 9 (1984), 3-35.

[4] P. L. Duren, Theory of $H^{p}$ Spaces, Academic Press, 1970.

[5] _- Univalent Functions, Springer-Verlag, New York, 1983.

[6] G. H. Hardy and J. E. Littlewood, Some properties of conjugate functions, J. Reine Angew. Math., 167 (193), 405-423. 
[7] T. H. MacGregor, Radial growth of a univalent function and its derivative off sets of measure zero, Contemporary Math., 38 (1985), 69-76.

[8] Chr. Pommerenke, Univalent Functions, Vandenhoeck and Ruprecht, Göttingen, 1975.

[9] M. Tsuji, Potential Theory, Chelsea Publishing Company, New York, 1959.

Received January 7, 1988 and in revised form October 11, 1988.

KuWAit UNIVERSITY

P.O. BoX 5969

13060-SaFAT-KuWAIT 


\section{PACIFIC JOURNAL OF MATHEMATICS EDITORS}

\author{
V. S. VARADARAJAN \\ (Managing Editor) \\ University of California \\ Los Angeles, CA 90024-1555-05 \\ Herbert Clemens \\ University of Utah \\ Salt Lake City, UT 84112 \\ ThOMAS ENRIGHT \\ University of California, San Diego \\ La Jolla, CA 92093
}

R. FINN

Stanford University

Stanford, CA 94305

HeRmann FlaschKa

University of Arizona

Tucson, AZ 85721

VAUGHAN F. R. JONES

University of California

Berkeley, CA 94720

Steven KerckHofF

Stanford University

Stanford, CA 94305
RoBION KIRBY

University of California

Berkeley, CA 94720

C. C. MOORE

University of California

Berkeley, CA 94720

Harold Stark

University of California, San Diego

La Jolla, CA 92093

\section{ASSOCIATE EDITORS}

\begin{tabular}{|c|c|c|c|c|}
\hline R. ARENS & $\begin{array}{l}\text { E. F. BECKENBACH } \\
(1906-1982)\end{array}$ & H. NeumanN & F. Wolf & K. YoshidA \\
\hline \multicolumn{5}{|c|}{ SUPPORTING INSTITUTIONS } \\
\hline \multicolumn{2}{|c|}{ UNIVERSITY OF ARIZONA } & \multicolumn{3}{|c|}{ UNIVERSITY OF OREGON } \\
\hline \multirow{2}{*}{\multicolumn{2}{|c|}{$\begin{array}{l}\text { UNIVERSITY OF BRITISH COLUMBIA } \\
\text { CALIFORNIA INSTITUTE OF TECHNOLOGY }\end{array}$}} & \multicolumn{3}{|c|}{ UNIVERSITY OF SOUTHERN CALIFORNIA } \\
\hline & & \multicolumn{3}{|c|}{ STANFORD UNIVERSITY } \\
\hline \multicolumn{2}{|c|}{ UNIVERSITY OF CALIFORNIA } & \multicolumn{3}{|c|}{ UNIVERSITY OF HAWAII } \\
\hline \multicolumn{2}{|c|}{ MONTANA STATE UNIVERSITY } & \multicolumn{3}{|c|}{ UNIVERSITY OF TOKYO } \\
\hline \multicolumn{2}{|c|}{ UNIVERSITY OF NEVADA, RENO } & \multicolumn{3}{|c|}{ UNIVERSITY OF UTAH } \\
\hline \multicolumn{2}{|c|}{ NEW MEXICO STATE UNIVERSITY } & \multicolumn{3}{|c|}{ WASHINGTON STATE UNIVERSITY } \\
\hline OREGON STA & UNIVERSITY & \multicolumn{3}{|c|}{ UNIVERSITY OF WASHINGTON } \\
\hline
\end{tabular}




\section{Pacific Journal of Mathematics}

\section{Vol. 141, No. $1 \quad$ November, 1990}

Yusuf Abu-Muhanna and Abdallah Khalil Lyzzaik, The boundary

behaviour of harmonic univalent maps $\ldots \ldots \ldots \ldots \ldots \ldots \ldots \ldots \ldots \ldots$

Lawrence Jay Corwin, Allen Moy and Paul J. Sally, Jr., Degrees and

formal degrees for division algebras and $\mathrm{GL}_{n}$ over a $p$-adic field $\ldots \ldots \ldots 21$

Ulrich Dierkes and Gerhard Huisken, The $n$-dimensional analogue of the

catenary: existence and nonexistence $\ldots \ldots \ldots \ldots \ldots \ldots \ldots \ldots 47$

Peter Gerard Dodds, C. B. Huijsmans and Bernardus de Pagter,

Characterizations of conditional expectation-type operators $\ldots \ldots \ldots \ldots 55$

Douglas Dokken and Robert Ellis, The Poisson flow associated with a

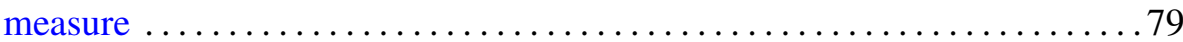

Larry J. Santoni, Horrocks' question for monomially graded modules . . . . 105

Zbigniew Slodkowski, Pseudoconvex classes of functions. II. Affine

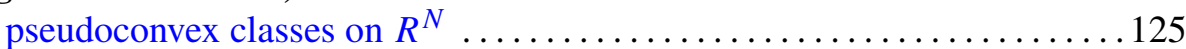

Dean Ellis Smith, On the Cohen-Macaulay property in commutative algebra

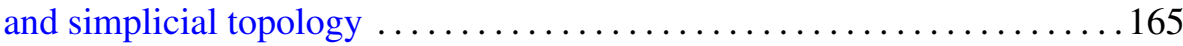

Michał Szurek and Jaroslaw Wisniewski, Fano bundles over $P^{3}$ and $Q_{3} \ldots 197$ 